\title{
FEED-FORWARD/FEEDBACK CONTROL FOR ACTIVE VIBRATION SUPPRESSION OF FLEXIBLE SPACECRAFT DURING ATTITUDE MANEUVER
}

\author{
Qinglei Hu${ }^{1}$, Guangfu Ma², Yaqiu Liü \\ ${ }^{1,2}$ School of Astronautics, Harbin Institute of Technology, Harbin 150001, China \\ ${ }^{3}$ School of Information and Computer Engineering, Northeast Forestry University
}

\begin{abstract}
This paper presents an investigation into development of feed-forward and feedback control strategies for active vibration suppression and attitude control of flexible spacecrafts. The feed-forward loop consists of a computed-torque scheme and a command shaping technique based on component synthesis vibration suppression (CSVS) method which was developed based on linear systems theory. For relaxing the requirements of dynamic linearity in traditional input component command in CSVS method, a new approach is developed for designing the input component command by utilizing a structure in which the modal forces of flexible modes vibration are shaped according to a predefined trajectory, which improves the performance of CSVS method in vibration suppression for the nonlinear attitude dynamics. This proposed control strategy is practical as it does not require direct measurement of flexible appendage vibration, and also has a simple structure with low on-line computational load. Numerical simulations demonstrate the effectiveness of the control strategy. Copyright (c) 2005 IFAC
\end{abstract}

Keywords: Active vibration suppression, Attitude maneuver, Feed-forward/feedback control, Flexible spacecraft.

\section{INTRODUCTION}

Modern spacecrafts often employ large, complex, and lightweight structures such as solar arrays, while requirement for attitude control performance becomes more stringent. For attitude operations that require small control actions, reaction/momentum wheels are used. However, during attitude maneuver, such as north-south station keeping and slew, the required torque are normally too high for reaction/momentum wheels. Therefore, thrusters are normally used for attitude control during these maneuvers. Nevertheless, the on-off thrusters produce nonlinear and discontinuous control actions, which may excite flexible modes of modern spacecraft. Designing an on-off control system to provide the pointing accuracy while avoiding interaction with the flexible structures poses a challenging task. Research toward thruster control has been focused on mainly two areas: bang-bang control and pulse modulation, see (Vander and $\mathrm{He}, 1983)$. Bang-bang control is simple in formulation, but results in excessive thruster action. On the other hand, pulse modulators are commonly employed due to their advantages of reduced propellant consumption and near-linear duty cycles. On-off thruster firing, no matter the method of modulation, will introduce vibrations to the flexible structures to some degree. Effectively suppressing the induced vibration poses a challenging task for spacecraft designers. One promising method for this problem is to modifying an existing command so that it results in less or zero residual vibration of a flexible spacecraft. Input shaping (Singer and Seering, 1990; Singhose and Seering, 1996) and CSVS (Liu, et al., 1988; Liu, et al., 1994; Shan, et al., 2004) are two commonly used methods to modify the input command to reduce vibrations of flexible structures. For the input shaping method, the input shaper is obtained by numerical value optimization so that the shaper is just the impulse sequences, see (Singer and Seering, 1990); while for CSVS method, it can have more forms of sequences not just impulse sequences by using analytical method, and the components can be any form.

CSVS method is a simple but effective feed-forward control scheme for vibration reduction of linear flexible systems. However, it should be noted that CSVS method is derived from the superposition 
principle of linear systems. When the system exhibits some nonlinear dynamics, the performance of CSVS method may be degraded and residual vibration will still exist. To overcome this drawback, in this paper, a new approach based on CSVS method is developed for designing the shaped component of the modal forces of flexible modes vibrations for compensating the nonlinear flexible dynamics system. It utilizes a structure in which the modal forces of modal vibrations are shaped through predefining a reference trajectory that can meet the vibration suppression target. This approach also relaxes the requirement of being a linear system in CSVS method. In order to follow the shaped reference trajectory identically or at least as closely as possible, a computed feed-forward torque control is placed inside the feed-forward loop. Simulation results of a rest-to-rest maneuver of a flexible spacecraft demonstrate the effectiveness of the control strategy.

\section{DYNAMICS AND MANEUVER DESCRIPTION}

Fig. 1 shows the model of a flexible spacecraft. The rotational motion only without any translation of the center of mass of the whole structure is considered in this paper. Define the OXY and oxy as the inertial frame and the frame fixed on the hub, respectively. The attitude angle $\theta$ denotes the relative motion between these two frames. Denote $w(x, t)$ as the flexible deformation at point $x$ with respect to the oxy frame. It is assumed that the control torque is applied to the rigid hub only.

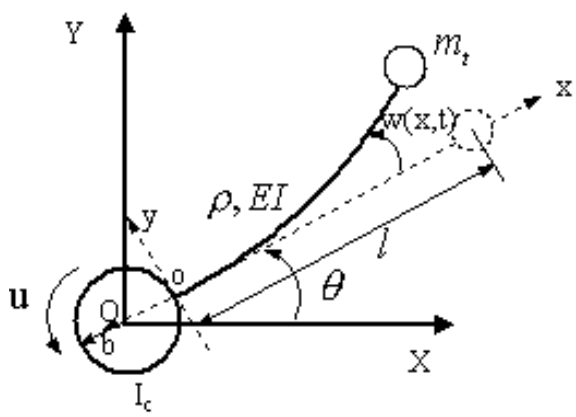

Fig.1 Spacecraft model with single-axis rotation

The governing equations of motion for the spacecraft model are given by, see (Liu, et al., 1994; Shan, et al., 2004)

$$
\begin{array}{r}
I_{c} \ddot{\theta}+2 \int_{0}^{l} \rho(x+b)\left((x+b) \ddot{\theta}+\frac{\partial^{2} w}{\partial t^{2}}\right) d x+2 m_{t} l\left((l+b) \ddot{\theta}+\left.\frac{\partial^{2} w}{\partial t^{2}}\right|_{l}\right)=u \\
\rho\left((x+b) \ddot{\theta}+\frac{\partial^{2} w}{\partial t^{2}}\right)+E I \frac{\partial^{4} w}{\partial x^{4}}=0
\end{array}
$$

where $I_{c}$ is the moment of inertia of the center body, $\rho$ is linear mass density of the appendage, $E I$ is the elastic rigidity of the flexible structure, $m_{t}$ is the tip mass, $b$ is the radius of center body, $l$ is the distance from the center origin to the tip of the flexible structure, and $u$ is the control torque applied by the actuator located at the center hub.
The flexible dynamics are also governed by the boundary conditions at the root and tip of the structure such as

$$
\begin{array}{ll}
w(x, t)=\frac{\partial w(x, t)}{\partial t}=0 & \text { at } x=0 \\
E I \frac{\partial^{2} w(x, t)}{\partial x^{2}}=0, & \\
E I \frac{\partial^{3} w(x, t)}{\partial x^{3}}=m_{t}\left(l \ddot{\theta}+\frac{\partial^{2} w(x, t)}{\partial t^{2}}\right) & \text { at } x=l
\end{array}
$$

The original hybrid differential equations of motion can be discretized into a finite dimensional mathematical model. Using the expansion method of unconstrainted modes series, the flexible displacement is approximated as

$$
w(x, t)=\sum_{i=1}^{N} \phi_{i}(x) \eta_{i}(t)
$$

where $\phi_{i}(x) \quad(i=1,2, \ldots, N)$ is the ith shape functions to be obtained by solving a characteristic equation for a cantilevered beam problem, $N$ elastic modes are considered and $\eta_{i}$ is the ith generalized coordinates for the flexible deflection.

The linearized second order matrix form of the differential equations of motion can be written as

$$
M \ddot{q}+K q=\left[\begin{array}{l}
1 \\
0
\end{array}\right] u
$$

where $M=\left[\begin{array}{cc}I_{\text {tot }} & M_{\theta \eta} \\ M_{\eta \theta} & M_{\eta \eta}\end{array}\right], K=\left[\begin{array}{cc}0 & 0 \\ 0 & K_{\eta \eta}\end{array}\right], q=\left[\begin{array}{l}\theta \\ \eta\end{array}\right]$ $\eta=\left[\eta_{1}, \eta_{2}, \ldots, \eta_{N}\right]^{r}$, and the moment of inertia of the whole structure, the element mass and stiffness matrices $\left(I_{\text {tot }}, M_{\theta_{\eta}}, M_{\eta \eta}, K_{\eta \eta}\right)$ are computed by using unconstrained mode equations.

\section{CONTROL STRATEGIES}

\subsection{PD Feedback Control}

In this section, PD control method is reinvestigated with a possible capability for the vibration suppression of flexible spacecraft. Considering the following PD control for the attitude control system

$$
u(t)=-K_{p}\left(\theta-\theta_{f}\right)-K_{d} \dot{\theta}
$$

where $\theta_{f}$ is the desired angle to be reached, the design parameters $K_{p}, K_{d}>0$.

Theorem Given the dynamics as described by equation (4), let the control law be computed as equation (5), then the equilibrium state of its closedloop system

$$
M \ddot{q}+K q=\left[\begin{array}{c}
-K_{p}\left(\theta-\theta_{f}\right)-K_{d} \dot{\theta} \\
0
\end{array}\right]
$$

is globally asymptotical stable, i.e., $\theta \rightarrow \theta_{f}$, and $\dot{\theta}, \eta, \dot{\eta} \rightarrow 0$ as time $t \rightarrow \infty$. 
Proof: Consider the energy-based Lyapunov function

$$
V=\frac{1}{2}\left[\begin{array}{l}
\dot{\theta} \\
\dot{\eta}
\end{array}\right]^{T} M\left[\begin{array}{l}
\dot{\theta} \\
\dot{\eta}
\end{array}\right]+\frac{1}{2} \eta^{T} K_{\eta \eta} \eta+\frac{1}{2} e^{T} K_{p} e \geq 0
$$

where $e=\theta-\theta_{f} . V=0$ only at the system equilibrium state, i.e., $\theta=\theta_{f}, \dot{\theta}=0$ and $\eta=\dot{\eta}=0$. The time derivative of Lyapunov function along the trajectories of the closed-loop system is

$$
\begin{aligned}
& \dot{V}=\left[\begin{array}{c}
\dot{\theta} \\
\dot{\eta}
\end{array}\right]^{T} M\left[\begin{array}{l}
\ddot{\theta} \\
\ddot{\eta}
\end{array}\right]+\dot{\eta}^{T} K_{\eta \eta} \eta+\dot{e}^{T} K_{p} e \\
& =-\dot{\theta}^{T}\left(K_{p} e+K_{d} \dot{e}\right)+\dot{e}^{T} K_{p} e=-\dot{\theta}^{T} K_{d} \dot{\theta}=-K_{d} \dot{\theta}^{2} \leq 0
\end{aligned}
$$

where $\dot{e}=\dot{\theta}-\dot{\theta}_{f}=\dot{\theta}$.

At this stage, it can only be concluded that under the PD control, the system energy does not increase. This is the so-called global stability in the sense of Lyapunov. To prove asymptotically stable, it has to be shown that $\dot{V}=0$ only at the system equilibrium state. As $K_{d}$ is positive, $\dot{V}=0$ only when $\dot{\theta}=0$. Invoking LaSalle (Khalil, 1992) invariance set theorem, one can see that the equilibrium state of the system is globally asymptotically stable.

Note that this PD control only feeds back the attitude angle $\theta$ and angular rate $\dot{\theta}$, and does not require any information of the modal vibration. It is a modelindependent feedback control and robustness against model uncertainties

\subsection{PD Feedback Control with Smooth trajectory}

Although the PD control seems simple and robust as neither the system's dynamics model is required nor is the modal vibration information fed back, this control strategy is of merely theoretical value for vibration suppression. This is because: a) the decaying speed of modal vibration slow as shown in the latter simulations, and b) more importantly, it may not be practical.

One intuitive approach to effectively eliminate the vibration is to seek a feedback system that, while providing the essential smooth controls, is primarily targeted to control the tip displacement of flexible spacecraft in a way that less vibration motion should be induced by the center torque input. Based on this idea, a modified PD (Junkins and Bang, 1991; Bang, et al., 1999) control can be obtained in which a smooth reference trajectory is generated a priori, and the corresponding stabilizing control law is designed so that the reference trajectory should be tracked by satisfying the tip displacement constrain equation. The required torque is calculated by regarding the flexible spacecraft as a rigid body, see (Junkins and Bang, 1991; Agrawal and Bang, 1995;)

$$
u(t)=u_{r}-K_{p}\left(\theta-\theta_{r}\right)-K_{d}\left(\dot{\theta}-\dot{\theta}_{r}\right)
$$

where the reference torque $u_{r}$ corresponds to a shaped bang-bang control torque profile. The mathematical expression of $u_{r}$ is given as

$$
u_{r}=I_{\text {tot }} \ddot{\theta}_{r}=N_{\text {max }} f(\Delta t, t)
$$

where the shaped torque profile function $f(\Delta t, t)$ is present in Fig.2, $N_{\max }$ is the magnitude of the maximum applied torque. $\theta_{r}$ is the reference trajectory, which can be built by the integration of the reference torque (8) twice.

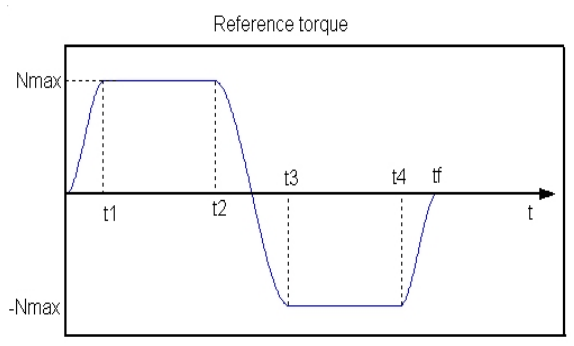

Fig.2 Shaped input function profile

Mathematically, it can also be expressed as (Bang, et al., 1999)

$$
f(\Delta t, t)\left\{\begin{array}{lr}
=\left(\frac{t}{\Delta \mathrm{t}}\right)^{2}\left[3-2\left(\frac{t}{\Delta t}\right)\right] & 0 \leq t \leq t_{1} \\
=1 & t_{1} \leq t \leq t_{2} \\
=1-2\left(\frac{t-t_{2}}{2 \Delta t}\right)^{2}\left[3-2\left(\frac{t-t_{2}}{2 \Delta t}\right)\right] & t_{2} \leq t \leq t_{3} \\
=-1 & t_{3} \leq t \leq t_{4} \\
=-1+\left(\frac{t-t_{4}}{\Delta t}\right)^{2}\left[3-2\left(\frac{t-t_{4}}{\Delta t}\right)\right] & t_{4} \leq t \leq t_{1}
\end{array}\right.
$$

where $t_{f}$ denotes the final maneuver time, and a useful relationship between the torque level and other parameters is derived as

$$
t_{f}=\sqrt{\frac{I_{t o t}\left(\theta_{f}-\theta_{0}\right)}{N_{\max }\left(1 / 4-\alpha / 2+\alpha^{2} / 10\right)}}
$$

The parameter $\alpha(0<\alpha<0.25)$ is a shaping parameter that determines the smoothness of the shaping action.

The time-varying control signal in equation (7) is now used as an argument of the sign function as

$$
u(t)=N_{\max } \operatorname{sign}\left(u_{r}-K_{p}\left(\theta-\theta_{r}\right)-K_{d}\left(\dot{\theta}-\dot{\theta}_{r}\right)\right.
$$

where sign is sign function. This control law produces a new switching function essentially different from that the strict minimum-time case and has some flexibility through selection of feedback gain $K_{p}, K_{d}$ to improve the slew maneuver angle response. However, the reference trajectory is extremely sensitive to variations in spacecraft parameters. Moreover, the original smoothing effect with continuously varying control output is not reflected in the switching function for thrusters.

\subsection{CSVS Method}


In this section, the principle of the CSVS method is discussed briefly only for the integrity of this paper. The following lemma gives the principle of component synthesis vibration suppression (CSVS) method to design the component sequences:

Lemma 1: Given a vibration mode with the natural frequency $\omega$, period $T_{d}$ and damping ratio $\varsigma$. Implementation of $n$ similar components, whose amplitudes are scaled by an attenuation factor of $e^{-\zeta \omega t}$ at $n$ time instants of $0, T_{d} / n, \cdots,(n-1) T_{d} / n$ leads to suppression of this vibration mode completely.

Ideally, all vibrations can be cancelled after applying the CSVS commands, provided that these two parameters can be known exactly. In practice, however, due to estimation errors of these two parameters, vibration may still exit after applying the CSVS commands. A robust CSVS method is proposed and fully analyzed by Shan, et al.(2004). The robustness can be recognized by the derivative of the system response with respect to the parameter.

Lemma 2: If a CSVS input command can suppress a vibration mode with the $p^{\text {th }}$-order robustness to the frequency estimation error, then the new command, formed by synthesizing $n$ of such commands according to Lemma1, can suppress the same vibration mode, but with the $\mathrm{p}^{+} 1^{\text {th }}$-order robustness to the frequency error.

The CSVS method can also work on suppression of multiple modes of vibrations. The principle of constructing the multi-mode CSVS commands is similar to that of constructing the robust CSVS commands. The following lemma 3 gives the principle of constructing the multi-mode CSVS commands:

Lemma 3: If a CSVS input command can suppress all the $m-1$ vibration modes, then the new command, formed by synthesizing $n$ of such commands according to Lemma 1 to suppress the $m^{\text {th }}$ vibration mode, can suppress all the $m$ vibration modes.

According to Lemma 1, Lemma 2, and Lemma 3, various CSVS commands can be constructed, with any number of components $n$, any order of robustness and any multiple modes.

Feed-forward/feedback control schemes. CSVS method is a simple but effective for vibration reduction of linear flexible systems. It should be noted that CSVS method is based on linear systems theory. When the system exhibits some nonlinear dynamics, the performance of CSVS method may be degraded and residual vibration will still exist in the flexible system. To overcome this drawback, modal forces of flexible modes vibration are used for preshaping.

Considering the lower part of Equation (5), it can be rearranged as

$$
M_{\eta \eta} \ddot{\eta}+K_{\eta \eta} \eta=-M_{\eta \theta} \ddot{\theta}
$$

where the left side is the modal vibration dynamics, and the right side defines the modal forces that are due to the coupling flexible and rigid dynamics. As $M_{\eta \eta}$ and $K_{\eta \eta}$ are time-invariant matrices, the modal forces are linear with respect to modal vibrations. Therefore, if we can have the modal forces shaped with CSVS method, then vibration free movement of the rigid body can be achieved. As the modal forces are functions of rigid body trajectories only, it is possible to predefine a reference trajectory that meets the above target. To implement such an idea, a new CSVS method structure is proposed as shown in Fig.3. In which $\theta_{0}$ and $\theta_{f}$ are the spacecraft initial and desired final angles, respectively. Given $\theta_{0}$ and $\theta_{f}$, the reference trajectory $\theta_{r}$ can be built by the integration of the reference torque (8) twice. Then the modal forces $M_{\eta \theta} \ddot{\theta}_{r}$ can be derived. The modal forces pass through CSVS structure for different modes and the shaped components $u_{s}$ in CSVS of the modal forces of flexible modes vibration are obtained. At the same time, the components in CSVS method can also be repeated for robustness. At the end, it is necessary to have an iteration loop to ensure that the spacecraft will stop at its desired angle within the desired settling time, i.e., $\theta_{s}\left(t_{f}\right)=\theta_{f}\left(t_{f}\right)$ and $\dot{\theta}_{s}\left(t_{f}\right)=0$, where $\theta_{s}$ is the shaped trajectory by CSVS method. In order to accomplish better control performance, the computed feed-forward torque control is used to follow the shaped reference trajectory identically or at least as closely as possible.

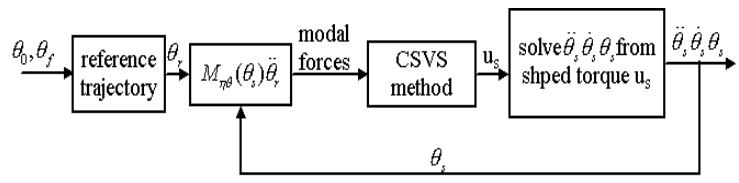

Fig.3 CSVS-based shaped modal forces

Combining a PD feedback control and regarding the spacecraft as rigid body, we have the following control law:

$$
u=I_{\text {tot }} \ddot{\theta}_{s}-K_{p}\left(\theta-\theta_{s}\right)-K_{d}\left(\dot{\theta}-\dot{\theta}_{s}\right)
$$

which is actually the same as Equation (8). Instead of using a smooth reference trajectory, the CSVS method is utilized to generate a more sophisticated rigid body trajectory. The control block diagram is shown in Fig.4.

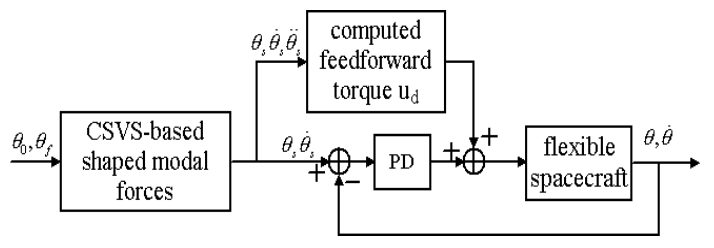

Fig.4 Feed-forward/feedback control structure 


\section{SIMULATION RESULTS}

The spacecraft simulation involves a single-axis rest-to-rest maneuver. The parameters for the simulated flexible spacecraft are given in the Ref. [7]. In this simulation, the flexible spacecraft is commanded to perform a $120^{\circ}$ slew. For comparative purposes, three different cases of a $120^{\circ}$ slew of the flexible spacecraft are conducted: (1) slew using the direct PD control as given in Section 3.1, (2) slew by applying the PD control along a smooth reference trajectory that is computed by a shaped bang-bang control torque profile, (3) slew using feedforward/feedback control based on shaped modal forces.

First, the PD control is employed in the attitude control to slew the flexible spacecraft for $120^{\circ}$. The angular displacement of the rigid body is shown in Fig.5 (a). Large overshoot of the slew angle resulted and the final desired angle was achieved after the maneuver. The vibrations of the first two flexible modes and control command are reflected in Figs.5 (b) and (c).

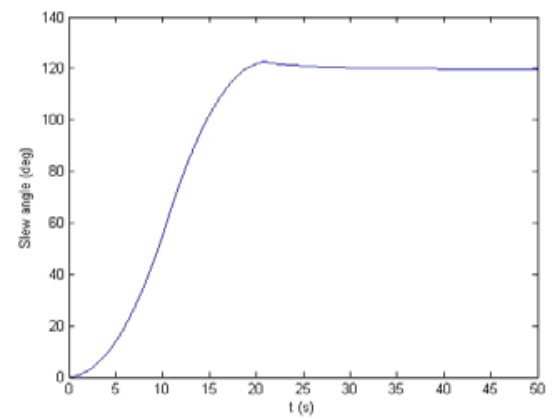

Fig.5 (a) Center body angle response with PD control
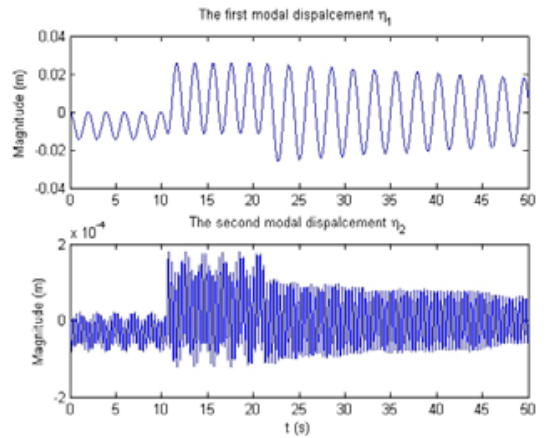

Fig.5 (b) Response of flexible modes with PD control

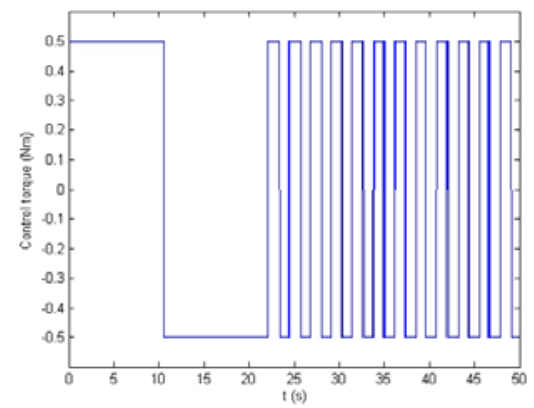

Fig.5 (c) Control command with PD control
Then the same control parameter is repeated with the PD control along a smooth reference trajectory. The results are shown in Fig.7. It is clear, from comparison of Fig.6 and Fig.7, the vibrations of the first two modes in Fig.7 (b) are smoother and less oscillatory than that in Fig.6 (b). This reflects the advantage of the case (2) over the case (1) for the vibration suppression. But there are a lot of control switches as shown in Fig.7 (c) and some little overshoot of the slew angle shown in Fig.7 (a).

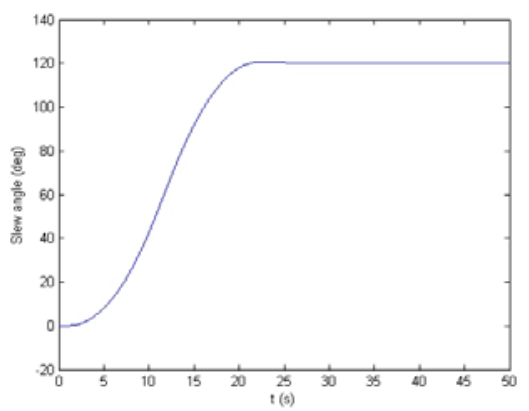

Fig.6 (a) Center body angle response with PD and smooth trajectory
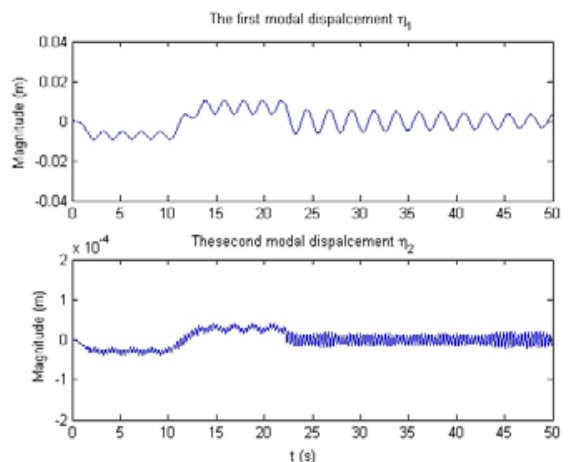

Fig.6 (b) Response of flexible modes with PD and smooth trajectory

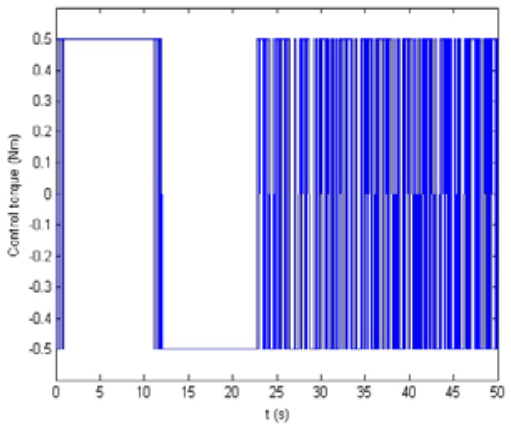

Fig.6 (c) Control command with PD and smooth trajectory

To demonstrate the improved performance of the CSVS method and to evaluate the capability of the control strategy working with the new components in the CSVS method, a $120^{\circ}$ slew of the flexible spacecraft using feed-forward/feedback control is conducted. The control parameters for the attitude control remain the same for a fair comparison, and the same reference trajectory with case (2) are used for the case (3). The system responses to the shaped modal forces are plotted in Fig.7. As compared with Fig.6, little vibrations of the first two flexible modes 
are observed, and there is no overshoot of the slew angle. This shows the effectiveness of the feedforward/feedback control based on shaped modal forces for active vibration suppression.

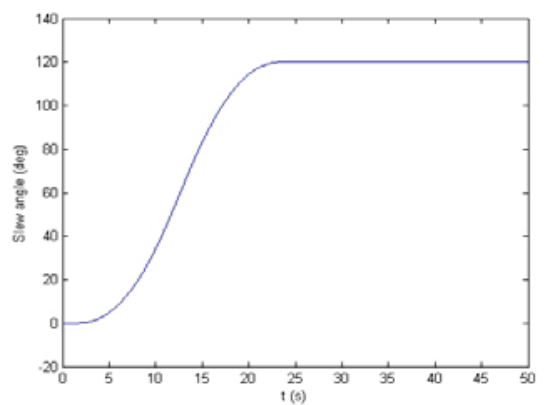

Fig.7 (a) Center body angle response with feedforward/feedback control
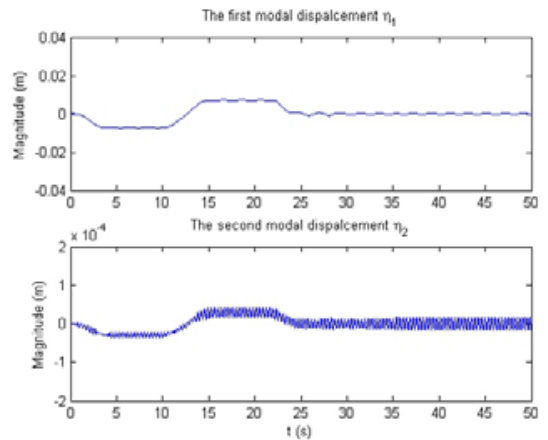

Fig.7 (b) Response of flexible modes with feedforward/feedback control

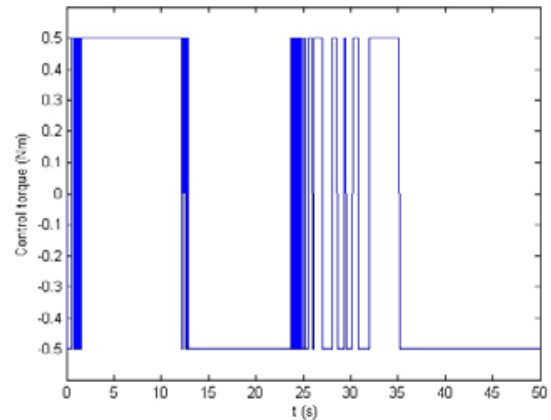

Fig.7 (c) Control command with feed-forward feedback control

\section{CONCLUSIONS}

The development of feed-forward and feedback control strategies for active vibration suppression and attitude control of a flexible spacecraft using modified CSVS method during attitude maneuver, respectively, have been proposed. A simple PD feedback control has been addressed to stabilize the attitude control system of flexible spacecraft. However, it is not effective in modal vibration suppression. Then, an attractively smooth approximation of sign function has been introduced to modify the admissible PD control law. Nevertheless, the trajectories are very sensitive to the variation in spacecraft parameter. Based on shaped modal forces of flexible modes vibration through predefining a reference trajectory that can meets the vibration suppression, a new modified CSVS method is developed for compensating for the nonlinear attitude dynamics. This approach relaxes the requirement of being a linear system in components design. To enhance the control robustness against model uncertainties, an independent feedback control is also used to further eliminate any residual vibration on the flexible appendage. As compared with these differences methods, performances of the techniques have been evaluated in terms of level of vibration reduction, speed of the response, and robustness through.

\section{REFERENCES}

Vander Velde, W. and He, J., (1983). Design of Space Structure Control Systems Using On-Off Thrusters. Journal of Guidance, Control, and Dynamics, Vol.6, No.1, pp.430-436.

Singer, N., and Seering, W., (1990). Preshaping Command Inputs to Reduce System Vibration. Transactions of the ASME Journal of Dynamic Systems, Measurement and Control, Vol.112, March, pp.76-82.

Singhose, W., Pao, L. and Seering, W., (1996). TimeOptimal Rest-to-Rest Slewing of multi-Modes Flexible Spacecraft Using ZVD Robustness Constraints. AIAA Paper, 96-3845, Aug.

D. Liu, D. M. Yang, J. Xi, and W. P. Zhang, (1988). An Optimal Maneuver Control Method for the Spacecraft with Flexible Appendage. AIAA/AAS Astrodynamics Conference, AIAA-88-4255, Aug. 15-17.

Liu, D. and Wu, G., (1994). An Active Vibration Suppression Method for the Flexible Spacecraft and its Application. AIAA/AAS Astrodynamics Conference, AIAA-1994-3757, Aug. 1-3, 1994.

Jin Jun, Shan, Dong Sun and Dun Liu (2004), Design for Robust Component Synthesis Vibration Suppression of Flexible Structures with On-off Actuators, IEEE Trans.Robotics and Automation, Vol.20,No.3, pp.512-525.

Khalil, H., (1992). Nonlinear system. New York: Macmillan.

Junkins, J. and Bang, H., (1991). Near-MinimumTime Control of Distributed Parameter System: Analytical and Experimental results. Journal of Guidance, Control, and Dynamics, Vol.14, No.2, pp.406-415.

Bang, H., Park, Y., and Han, J., (1999). Feedback control for slew maneuver using on-off thrusters. Journal of Guidance, Control, and Dynamics, Vol.22, No.6, pp.816-822.

Agrawal, B., and Bang, H., (1995). Robust Closedloop control design for spacecraft maneuver using on-off thrusters. Journal of Guidance, Control, and Dynamics, Vol.18, No.6, pp.13361349. 